\title{
THE RR LYRAE STARS IN THE MAGELLANIC CLOUDS
}

\author{
J. A. GRAHAM \\ Cerro Tololo Inter-American Observatory*
}

\begin{abstract}
The characteristics of the RR Lyrae stars in the Magellanic Clouds are discussed. The existence of numerous RR Lyrae variables in the Large Magellanic Cloud (LMC) cluster NGC 1835 is noted. The variables indicate that this cluster is of Oosterhoff type I. No cluster of Oosterhoff type II has yet been recognized in the Clouds. Some new results of a study of the RRLyrae variables in an LMC field $1^{\circ} \times 1.3$ are discussed. Periods have been determined for 50 out of 72 probable RR lyraes in this field. RR Lyrae $a b$ stars with periods less than 0.46 are absent. The time averaged $\overline{\langle B\rangle}$ and $\overline{\langle V\rangle}$ are $19 m .56$ and $19 m .20$ with surprisingly small dispersion. First impressions of results for a similar field in the Small Magellanic Cloud (SMC) suggest that the old stellar population of the SMC may have a slightly lower metal abundance than that of the LMC. The best available distance moduli for the Magellanic Clouds indicate a mean absolute visual magnitude $M_{\langle\bar{v}\rangle}$ of $+0 \mathrm{~m} .5 \pm 0.2$ for the RR Lyrae stars in these systems.
\end{abstract}

\section{Introduction}

When we come to study the older populations of the Magellanic Clouds, we are no longer very concerned with the well-known spectacular features which dominate most photographs of these stellar systems. We must look past the brilliant associations with their blue supergiants and $\mathrm{H}$ II regions, past the Cepheid variables and the numerous open star clusters until we see in each Cloud only the faint amorphous substrata which are made up of stars and planetary nebulae with ages $10^{9} \mathrm{yr}$ or more. Relieving the general uniformity, several old globular clusters, similar to those of our own Galaxy are seen, scattered among the two Clouds. Except for the occasional nova every star in this old population is fainter than 15th magnitude. However, despite their rather dull appearance, these faint substrata of low mass stars are indeed the structural backbones of the Magellanic Clouds. They probably contain a significant fraction of the mass of each system and thereby are important contributors to the internal dynamics of the Clouds. It is also important to realise that the study of these old populations may give information about the history of nuclear synthesis in the Clouds back to the earliest stages of their evolution.

Detailed investigation of the faint Population II stars of the Magellanic Clouds will have to wait for the new 4-m reflectors to begin work in the Southern Hemisphere. However, quite a bit of work can be done now. In a recent review article, Westerlund (1970) has discussed much of our present knowledge of the Cloud populations. The planetary nebulae have been studied in considerable detail by Feast (1968), Webster (1969), and Smith and Weedman (1972). Novae are being detected at the rate of 1 or 2 a year at Cerro Tololo Inter-American Observatory by Graham and Araya (1971). Gascoigne's (1966) investigation of the color-magnitude diagrams of the Cloud globular clusters has recently been extended by Walker (1970, 1971, 1972),

* Operated by the Association of Universities for Research in Astronomy, Inc., under contract with the National Science Foundation. 
who has made use of electronographic techniques to reach stars as faint as blue magnitude 23.5. In the present article, the characteristics of the RR Lyrae-type variables are described. These stars are perhaps the easiest of the Population II objects to discover because of the short period and substantial amplitude of their light variation. In the context of this colloquium, I think that it is important to stress that not only can the RR Lyraes tell us about the Magellanic Clouds, but that also the Magellanic Clouds can tell us quite a lot about RR Lyrae stars. Among the globular clusters and related systems that we are concerned with at this conference, the Magellanic Clouds alone offer the opportunity to compare, at effectively the same distance and often on the same photographic plate, an abundance of the youngest Population I and the oldest Population II stars.

RR Lyrae stars were first detected in the Magellanic Clouds by Thackeray (1951, 1958) who found three in the Small Magellanic Cloud (SMC) cluster NGC 121 and three in the surrounding field outside the probable boundary of the cluster. Thackeray also discovered RR Lyraes in the Large Magellanic Cloud (LMC) globular clusters NGC 1978 (Thackeray, 1951), NGC 1466 (Thackeray and Wesselink, 1953), and later in NGC 2257 (Alexander, 1960). Further photometry of these variables has been carried out by Alexander (1960), Tifft (1963), Gascoigne (1966), Wesselink (1971), and Norris (1972).

\section{Cerro Tololo Investigations}

The recent availability of large reflecting telescopes with wide photographic fields has made much easier the discovery of the RR Lyrae variables in the general field of the Magellanic Clouds. With the CTIO 1.5-m reflector, stars of blue magnitude 20.5 can be recorded with a $30-\mathrm{min}$ exposure over a field of 1.5 on $103 \mathrm{a}-\mathrm{O}$ plates. The $f / 7.5$ scale of the telescope is $18.1^{\prime \prime} \mathrm{mm}^{-1}$. With $20 \mathrm{~cm} \times 25 \mathrm{~cm}$ plates an effective field of $1^{\circ} \times 1.3$ is obtained. Vignetting appears to be less than $0^{\mathrm{m}} .1$ over this area.

There are four principal fields for which series of plates are available. One field is centered on the LMC cluster NGC 1783. This region was chosen because it contains the photoelectric sequence determined by Gascoigne (1962). A second field, in the vicinity of the LMC bar, is between the globular cluster NGC 1835 and the photoelectric sequence of Tifft and Snell (1971). In the SMC, many plates have now been taken of a field centered on the globular cluster NGC 121. Tifft (1963) has published a sequence going to $B=20.8$ close to this cluster. Finally, some plates have been taken of a region closer to the SMC bar. These are centered between NGC 361 and NGC 362. 103a-O plates were initially used to obtain the maximum number of plates and the greatest time resolution. However, since it became clear that precise blue magnitudes are very important, longer exposures with $103 a-O+G G 13$ filter have been taken. For each field two or three yellow sensitive plates (103a-D + GG 14) have been obtained. These are useful for the determination of mean $\mathrm{V}$ magnitudes for the RR Lyrae variables, as well as for the identification of very red stars in the field.

The variables are discovered by comparing pairs of plates, taken under similar conditions a week or more apart. For each field it is planned eventually to compare 
10 such pairs. Following the discovery, the variables and standard sequence stars are measured on each plate with an iris-diaphragm photometer. The transformation curve relating iris-diaphragm reading and magnitude is determined by eye estimate only.

\section{RR Lyrae Stars in the Magellanic Cloud Globular Clusters}

To the body of work already published, I am able to add some new data for the variables in an additional cluster, NGC 1835. Sra. Maria Teresa Ruiz, a former student at the University of Chile, has helped me considerably in this part of the investigation. NGC 1835 is a very compact cluster quite close to the LMC bar. Because of crowding both in the cluster and in the surrounding dense star field, we have only been able to use plates taken under the best seeing conditions. Although much of the cluster is unresolved, it is clear that it is very rich in variables. Forty-nine stars were suspected of variation from the blink comparisons. Subsequent iris-diaphragm photometer measurements have shown that for twenty-one of the forty-nine, the variation is certainly real. Periods have been found for ten stars. Nine of these are RR Lyrae $a b$-type stars, and one a $c$-type. Among the eleven cases remaining, there is one long period variable, one possible Cepheid and a few more RR $c$-type variables. The standard magnitude sequence for this cluster is calibrated in two ways: first by a photographic transfer from the Gascoigne (1962) NGC 1783 sequence and second by direct measurement of the nearby Tifft-Snell sequence on the same plate. Agreement is found within 0 m.1 for the stars calibrated in both ways.

In Table I, I summarise the presently available data for the Cloud cluster RR Lyraes. The arithmetic mean of the $a b$-type variable star periods, the arithmetic means of the apparent blue median magnitude, of the time-averaged blue magnitudes

TABLE I

RR Lyraes in Magellanic Cloud globular clusters

\begin{tabular}{|c|c|c|c|c|c|c|c|}
\hline \multicolumn{2}{|c|}{ Cluster } & \multirow{2}{*}{$\begin{array}{l}\begin{array}{l}\text { No. of variables } \\
\text { with periods }\end{array} \\
3\end{array}$} & \multirow{2}{*}{$\begin{array}{c}\bar{P}_{a b} \\
0.560\end{array}$} & \multirow{2}{*}{$\frac{\bar{B}_{\text {med }}}{19 m 55}$} & \multirow{2}{*}{$\begin{array}{l}\overline{\langle B\rangle} \\
19 m 7\end{array}$} & \multirow{2}{*}{$\begin{array}{l}V_{\mathrm{RG}} \\
16 \mathrm{~m} \cdot 7\end{array}$} & \multirow{2}{*}{$\begin{array}{l}\text { Ref. } \\
(1)\end{array}$} \\
\hline SMC & 121 & & & & & & \\
\hline & $(1466$ & 10 & 0.549 & & 19.23 & 16.2 & $(2)(3)(4)$ \\
\hline \multirow[t]{3}{*}{ LMC } & $\{1835$ & 10 & 0.572 & 19.25 & 19.40 & 16.0 & (5) \\
\hline & 2257 & 6 & 0.558 & 19.19 & 19.31 & 16.1 & (2) (6) \\
\hline & & & 0.559 & 19.22 & 19.31 & 16.1 & \\
\hline
\end{tabular}

\section{References:}

(1) Tifft (1963).

(2) Gascoigne (1966).

(3) Wesselink (1971).

(4) Norris (1972).

(5) Graham and Ruiz (unpublished).

(6) Alexander (1960). 
and of the apparent visual magnitude at the red giant branch tip are given where possible for each cluster. One notes immediately that all the above clusters are of Oosterhoff type I on the basis of the variable star data. Although the numbers are still small, it seems possible that Oosterhoff type II clusters may turn out to be rare or absent in the Magellanic Clouds, indicative perhaps of the absence of an extremely metal poor population. A similar suggestion comes from the work of Andrews and Lloyd Evans (1971) who have found spectra indicating only moderate metal deficiency for a sample of thirteen Magellanic Cloud clusters, In Table I, it should also be noted, a comparison between the three LMC globulars and the one SMC globular suggests that the SMC has an apparent distance modulus $0.4 \pm 0.2$ greater than that of the LMC.

\section{The Field RR Lyrae Stars in the Magellanic Clouds}

For the four fields mentioned in Section 2, only the analysis for the NGC 1783 field has progressed sufficiently so that good data are now available for the variables in this field. This region of the LMC is of special interest as it contains not only the intermediate age cluster NGC 1783 but also a strong Population I component in the form of numerous Cepheids, blue supergiants and prominent $\mathrm{H}$ II regions. Clearly, in this part of the LMC we are viewing stars with a very wide range in age. To detect the RR Lyrae stars, ten pairs of plates were blinked and 125 stars marked as suspected variables. Of these, only one, an eclipsing variable, is a possible member of NGC 1783 itself (Graham, 1970). The iris-diaphragm measurements indicate that about 50 are Cepheid, red, or eclipsing variables, 72 are probable RR Lyrae stars. Periods have been determined for 51 of the 72 suspected RR Lyraes. Only one of the 51 is a $c$-type variable, although a few of the 21 difficult cases may also be RR Lyraes of this type. There seems little doubt, however, that the $c$-type variables are relatively rare compared to the $a b$-type.

Table II summarises the characteristics of the NGC 1783 sample. Note the absence of the $\mathrm{RR}_{a b}$ stars with periods less than 0.46 , which form about $25 \%$ of the $\mathrm{RR}_{a b}$ population in the vicinity of the Sun.

TABLE II

RR Lyrae stars in the NGC 1783 field in the LMC

Number of probable RR Lyraes $\quad 72$

Number of RR $\mathbf{R}_{a b}$ stars with periods $\quad 50$

Number of $R_{R_{c}}$ stars with periods 1

Most frequent period between 0.50 and 0.55 .

$\mathbf{R R}_{a b}$ stars with $P<0$ d 46 absent.

Period-amplitude diagram similar to that of

Messier 3 but with more dispersion.

$$
\begin{aligned}
\bar{B}_{\text {median }} & =19 \text { m.35 } \\
\overline{\langle\boldsymbol{B}\rangle} & =19 \text { m56 } \\
\overline{\langle V\rangle} & =19 \mathrm{~m} .20
\end{aligned}
$$


The mean magnitudes given in Table II are a tenth of a magnitude or so fainter than the cluster values in Table I. A similar result was found by Tifft in a comparison between the RR Lyraes in the SMC cluster NGC 121 and those in the general field. It seems likely that this effect is largely due to background enhancement arising from faint, unresolved stars and from light scattered by the bright cluster center, and that the values derived from field star observations are the more accurate ones.

A surprising result of the present investigation is the small dispersion in the time averaged blue magnitude, $\langle B\rangle$, among the LMC RR Lyraes. In Figure 1, two histograms are presented comparing the dispersion of the NGC 1783 field variables and of the Messier 3 (M3) variables (Sandage, 1959). It should be noted that the mean magnitudes of the M3 variables are intensity means, while those presently available for the LMC variables are time-averaged magnitudes. However, this should not affect the present comparison. The dispersion among the Cloud RR Lyraes appears to be only slightly greater than that found among the M3 stars. Besides intrinsic dispersion, the dispersion among the Cloud sample must include such factors as interstellar absorption within the Cloud, scatter in distance along the line of sight (at the LMC distance

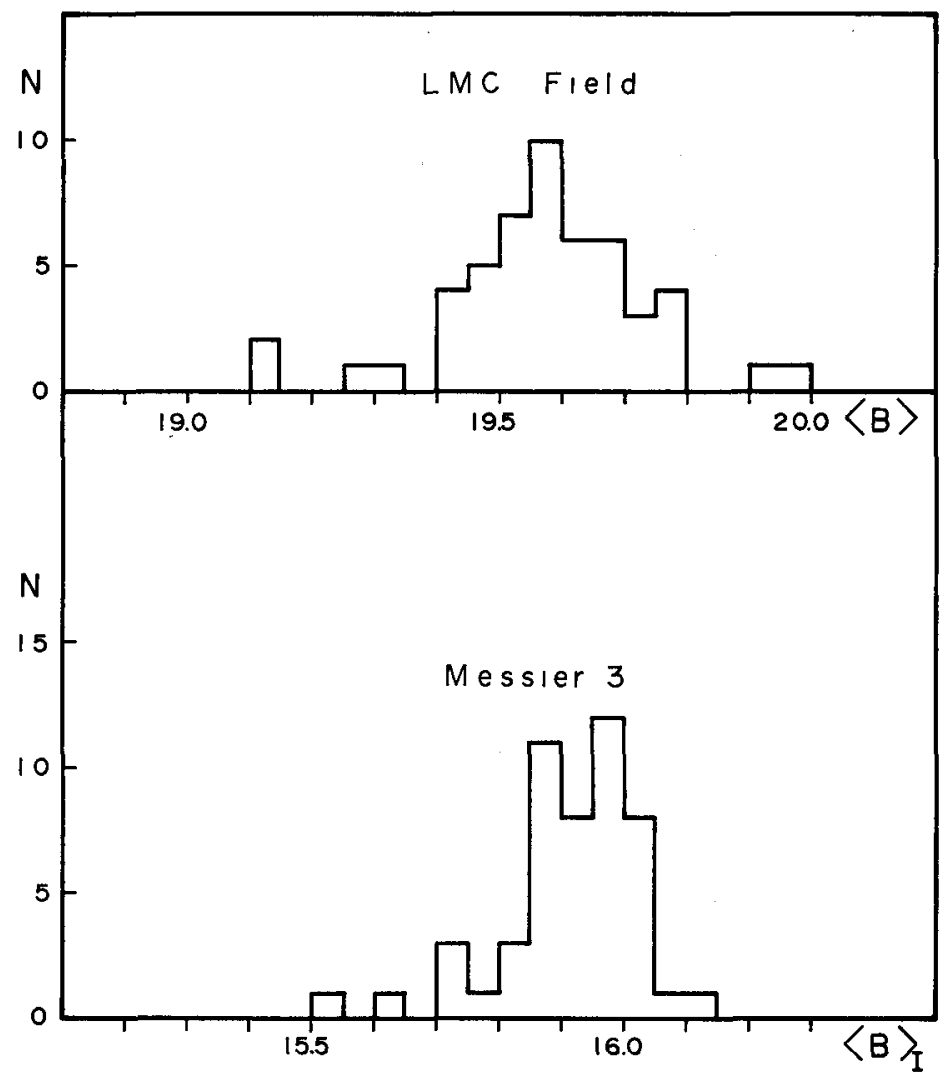

Fig. 1. Frequency distribution of the apparent blue magnitudes of the RR Lyrae stars in the LMC field near NGC 1783 and of the RR Lyrae stars in the Galactic globular cluster Messier 3. 
$\left.\pm 1 \mathrm{kpc}= \pm 0^{\mathrm{m}} .04\right)$ as well as photographic plate errors. The small dispersion found indicates that (1) the intrinsic dispersion of the RR Lyrae absolute magnitudes is small, not exceeding the dispersion found among the M3 variables by more than $0 \% 1$ and (2) probably the Cloud RR Lyraes are concentrated in a disk system rather than spread through an extended halo system. The Christy pulsation theory (summarised by Christy (1966)) predicts that in the Galaxy the observed range in metal abundance will produce a range in absolute magnitude for the field RR Lyraes of about 0.4 . The low dispersion found for the absølute magnitudes of the LMC variables suggests that if this theory is correct, the range of metal abundance may be quite small among the old Cloud population. Some support for this suggestion comes from the observed absence of the short period $\mathrm{RR}_{a b}$ variables with $P<0$ d 46 days and, at the other extreme, by the possible absence of Oosterhoff type II globular clusters with weak-lined integrated spectra.

It is possible to obtain first impressions of the characteristics of the RR Lyraes in the SMC using some measurements of the available plate material for the NGC 121 field. More plates are still required before a detailed analysis can be undertaken. Table III summarises the SMC situation at present. If the first impressions are correct, then from comparing Tables II and III, there do seem to be some significant differences between the RR Lyraes in the two Magellanic Clouds.

\section{TABLE III}

First impressions of the RR Lyrae stars in the NGC 121 field in the SMC

\begin{tabular}{lr}
\hline Number of Probable RR Lyraes & 60 \\
Number of $\mathrm{RR}_{a b}$ stars with periods & 16 \\
Number of $\mathrm{RR}_{c}$ stars with periods & 4 \\
Most frequent period between 0.55 and $0 \mathrm{~d} 60$ \\
$\operatorname{RR}_{a b}$ stars with $P<0$ d50 rare or absent. \\
$\qquad \vec{B}_{\text {medan }} \approx 19 \mathrm{~m} .8$ \\
$\frac{\langle B\rangle}{\langle V\rangle} \approx 20$ m 0 \\
\hline
\end{tabular}

In the context of the Christy theory, the transition point between the fundamental and first overtone modes of pulsation seems to be shifted relative to the LMC by about 0.05 towards a longer period in the case of the SMC. This may indicate that the old population in the SMC is slightly metal poor, on the average, compared with that of the LMC. The values for the mean magnitudes are still uncertain, and, considering the preliminary state of the analysis, the agreement with the earlier work of Tifft (1963) is quite reasonable.

\section{The Absolute Magnitudes of the Magellanic Cloud RR Lyrae Stars}

The distances of the Magellanic Clouds are now sufficiently well known for it to be possible to make a good estimate of the absolute magnitude of their RR Lyrae vari- 
ables. Table IV summarises what are probably the best available determinations. In making the interstellar absorption corrections for this compilation, I am assuming that there may be appreciable absorption within the Cloud Population I component but that outside of this, there is negligible absorption between the Magellanic Clouds and the Sun. This may be an oversimplification but I am impressed by some recent work of Philip (1971) which shows the effective absence of interstellar reddening in

TABLE IV

The distance moduli of the Magellanic $\dot{C}$ louds

\begin{tabular}{lll}
\hline & LMC & SMC \\
\hline Cepheids (observed) & $18^{\mathrm{m}} \cdot 6$ & 19.3 \\
Cepheids (theory) & 18.7 & 19.1 \\
Globular clusters & 18.7 & 19.2 \\
Old Population & 18.6 & 18.9 \\
Novae (half weight) & $\underline{19.0}$ & $\underline{19.4}$ \\
Average & $\underline{18.7}$ & $\underline{19.2}$ \\
$\langle V\rangle$ RR Lyraes $=$ & 19.2 & 19.6 \\
$M\langle\bar{V}\rangle$ RR Lyraes $=$ & $+0^{\mathrm{m}} 5$ & $+0^{\mathrm{m}} 4$ \\
\hline
\end{tabular}

front of an open cluster at a distance of $360 \mathrm{kpc}$ along the line of sight to the LMC and also by the remarks of Gascoigne (1969) concerning the reddening of the SMC. In Table IV, two determinations of Cloud moduli from Cepheids are included, one set depending on the very comprehensive discussions of the observational data by Sandage and Tammann (1971) and by Gascoigne (1969), and another set depending on the theoretical interpretation by Christy (1971) of the observed Cepheid light curves. The values of the Cloud moduli due to the globular cluster observations come from Gascoigne (1966) and include a small correction to take into account new observed distance moduli by Sandage (1970) for some Galactic globular clusters. The differential modulus given in Section 2 of this paper is used to calculate the corresponding SMC distance modulus. The values for the old population are taken from Tifft (1963) and Tifft and Snell (1971). The determinations from novae (van den Bergh, 1968) are given only half weight as they presently depend on incomplete observations of only a few objects. Hopefully, this situation will be improved now that novae are being more systematically detected. In compiling Table IV, care has been taken to avoid any determination which is based on an assumed absolute magnitude for the RR Lyrae stars. After averaging all results arithmetically, the adopted true moduli are $18^{\mathrm{m}} .7$ for the LMC and $19^{\mathrm{m}} .2$ for the SMC. Internal consistency suggests that each of these is uncertain by $\pm 0^{\mathrm{m}} \cdot 1$, provided that the assumptions about interstellar absorption are correct.

Using these distance moduli, the time-averaged visual magnitudes for the Cloud RR Lyraes come out to be $+0{ }^{\mathrm{m}} 5 \pm 0.2$ for the $\mathrm{LMC}$ and $+0{ }^{\mathrm{m}} 4 \pm 0.3$ for the SMC. In many respects (e.g. light curve shape, period distribution, amplitude of light variation), the Magellanic Cloud RR Lyraes seem to be very similar to those in our Galaxy and 
it is no surprise that the new absolute magnitudes agree well with most other determinations, such as the recent one by Sandage (1970). The fainter values suggested by Clube (1971) for metal-poor RR Lyraes in the Galaxy are certainly not supported by the present investigation.

\section{Acknowledgements}

I would like to express my gratitute to Sr Luis Elicer who has helped me with the iris photometry. Thanks are also due to several colleagues at Cerro Tololo Inter-American Observatory and at Steward Observatory for their comments on an early version of this manuscript.

\section{References}

Alexander, J. B.: 1960, Monthly Notices Roy. Astron. Soc. 121, 97.

Andrews, P. J. and Lloyd Evans, T.: 1971, in A. B. Muller, (ed.), The Magellanic Clouds, D. Reidel, Dordrecht, Holland, p. 88.

Bergh, S. van den: 1968, Comm. David Dunlap Obs. No. 195.

Christy, R. F.: 1966, Ann. Rev. Astron. Astrophys. 4, 353.

Christy, R. F.: 1971, in A. B. Muller (ed.), The Magellanic Clouds, D. Reidel, Dordrecht, Holland, p. 136

Clube, S. V.: 1971, in C. de Jager (ed.), Highlights of Astronomy (XIV General Assembly IAU), D. Reidel, Dordrecht, Holland, p. 788.

Feast, M. W.: 1968, Monthly Notices Roy. Astron. Soc. 140, 345.

Gascoigne, S. C. B.: 1962, Monthly Notices Roy. Astron. Soc. 124, 201.

Gascoigne, S. C. B.: 1966, Monthly Notices Roy. Astron. Soc. 134, 59.

Gascoigne, S. C. B.: 1969, Monthly Notices Roy. Astron. Soc. 146, 1.

Graham, J. A.: 1970, Publ. Astron. Soc. Pacific 82, 918.

Graham, J. A. and Araya, G.: 1971, Astron. J. 76, 768.

Norris, M. V.: 1972, in IAU Colloquium No. 21.

Philip, A. G. D.: 1971, Bull. Am. Astron. Soc. 3, 367.

Sandage, A.: 1959, Astrophys. J. 129, 596.

Sandage, A.: 1970, Astrophys. J. 162, 841.

Sandage, A. and Tammann, G. A.: 1971, Astrophys. J. 167, 293.

Smith, M. G. and Weedman, D. W.: 1972, (in press).

Thackeray, A. D.: 1951, Observatory 71, 219.

Thackeray, A. D.: 1958, Monthly Notices Roy. Astron. Soc. 118, 117.

Thackeray, A. D. and Wesselink, A. J.: 1953, Nature 171, 693.

Tifft, W. G.: 1963, Monthly Notices Roy. Astron. Soc. 125, 199.

Tifft, W. G. and Snell, C.: 1971, Monthly Notices Roy. Astron. Soc. 151, 365.

Walker, M. F.: 1970, Astrophys. J. 161, 835.

Walker, M. F.: 1971, Astrophys. J. 167, 1.

Walker, M. F.: 1972, Monthly Notices Roy. Astron. Soc. (in press).

Webster, B. L.: 1969, Monthly Notices Roy. Astron. Soc. 143, 97.

Wesselink, A. J.: 1971, Monthly Notices Roy. Astron. Soc. 152, 159.

Westerlund, B.E.: 1970, in A. Beer (ed.), Vistas in Astronomy 12, Pergamon Press, New York, p. 335.

\section{DISCUSSION}

Wesselink: Did you compare your distance modulus of NGC 121 with the one derived by Tifft? Graham: Yes, it agrees well.

Belserene: If Clube is right about the faintness of the RR Lyr stars, wouldn't that require a change in the distance scale? You would have smaller moduli for the Clouds and would recover fainter magnitudes for your RR Lyraes in agreement with his. 
Graham: It is hard to believe that the errors involving the Magellanic Cloud distance moduli can be large enough for this to be true.

Buscombe: Are the blue globular clusters of the LMC related to old or young populations?

Evans: Andrews and I have measured radial velocities of 14 of these clusters and find the velocity dispersion is not different from that of the entire Population I.

Buscombe: Are the 'blue globulars' kinematically related to Population II, or merely very rich open clusters?

Graham: Holland Ford in his thesis found, I believe, that they had Population I characteristics. Cooper: I believe Soviet work has determined some absolute magnitudes for RR Lyrae stars to be considerably closer to 0.0 than 0.5 . Can you comment on this?

Graham: I am not aware of these results. 